Cahiers d'études africaines

$240 \mid 2020$

Soudan : identités en tension

\title{
Verhoeven Harry. - Water, Civilization and Power in Sudan
}

Raphaëlle Chevrillon-Guibert

\section{OpenEdition}

Journals

Édition électronique

URL : https://journals.openedition.org/etudesafricaines/32988

DOI : 10.4000/etudesafricaines.32988

ISSN : 1777-5353

\section{Éditeur}

Éditions de l'EHESS

\section{Édition imprimée}

Date de publication : 2 décembre 2020

Pagination : 1045-1047

ISSN : 0008-0055

\section{Référence électronique}

Raphaëlle Chevrillon-Guibert, «Verhoeven Harry. - Water, Civilization and Power in Sudan », Cahiers d'études africaines [En ligne], 240 | 2020, mis en ligne le 02 décembre 2020, consulté le 04 janvier 2023. URL : http://journals.openedition.org/etudesafricaines/32988 ; DOI : https://doi.org/10.4000/ etudesafricaines.32988

Ce document a été généré automatiquement le 4 janvier 2023

Tous droits réservés 


\title{
VERHOEVEN Harry. - Water, Civilization and Power in Sudan
}

\author{
Raphaëlle Chevrillon-Guibert
}

\section{RÉFÉRENCE}

VerhoeVen Harry. - Water, Civilization and Power in Sudan. Cambridge, Cambridge

University Press, 2015, 338 p., bibl., index, ill.

1 Issu de son travail de thèse soutenu en 2012, l'ouvrage de Harry Verhoeven entend étudier les efforts du régime militaro-islamiste, arrivé au pouvoir au Soudan en 1989 et auto-baptisé Al-Ingaz pour transformer l'économie politique du pays. Il montre combien le projet de «salut économique » est au cœur du projet islamiste et mérite en cela une attention particulière pour qui souhaite comprendre le fonctionnement du régime. L'auteur se concentre exclusivement sur l'un des aspects de ce salut économique: sa «mission hydro-agricole» qui consiste en l'érection de grands ouvrages sur le Nil qui permettront l'irrigation de larges parcelles de terre et leur exploitation intensive. Si cette mission a été pensée dès la prise de pouvoir des islamistes, celle-ci n'a pu se réaliser qu'à la faveur du développement de l'économie pétrolière des années 2000 et de l'éviction de Hassan Al-Tourabi et de ses fidèles. Verhoeven interroge tout au long de l'ouvrage les manières de faire des dirigeants et leurs résultats quant à cette mission, et le verdict est sans appel : bien que la mise en œuvre de ce projet pharaonique ait été rendue possible par les pétrodollars et une scène politique régionale et internationale renouvelée, le développement tant espéré est loin d'être au rendez-vous. Au final, les islamistes n'ont fait que reproduire la violence de l'économie politique des précédents régimes alliant corruption, patronage et autoritarisme, mais aussi un fort désintérêt pour les régions périphériques pourtant exploitées dans ce type de projet.

2 Si à travers l'étude de cette mission, l'ouvrage entend tout particulièrement éclairer les ressorts du régime d'Al-Ingaz dans sa seconde décennie de pouvoir, il le fait en mettant 
le focus sur les élites politiques qui composent ce régime, leurs relations, discours et stratégies évoluant continuellement en fonction des contextes nationaux et internationaux, dans une étude de science politique somme toute très classique où peu d'attention est portée au local et à la façon dont les politiques économiques et leurs instruments fonctionnent concrètement. En ce sens, la façon dont le travail de terrain approfondi de l'auteur est présenté s'avère parlante: ce dernier cite les nombreux entretiens qu'il a menés avec des élites politiques ou administratives et celles-ci ont chaque fois été interrogées individuellement tandis qu'il n'invoque les individus ordinaires impliqués dans cette mission à différents titres (notamment comme citoyens déplacés par les projets de barrages ou expropriés) que de façon collective et très ponctuelle. Un second point qui fait défaut à la démonstration consiste en l'occultation quasi totale de la problématique de la région du Darfour et des Darfouriens qui étaient pourtant initialement nombreux à rejoindre le mouvement islamique soudanais. L'auteur pointe avec justesse combien le projet de construction de barrages tout comme celui de promotion d'une agriculture irriguée intensive qui lui est lié a favorisé un certain groupe, politisé et issu de quelques tribus de la vallée du Nil, mais il est muet sur les mécanismes concrets, et notamment économiques, qui ont exclu les islamistes darfouriens, un aspect pourtant central de la transformation du régime d'Al-Ingaz que traduit notamment l'exclusion de Tourabi. Quoiqu'il en soit, l'ouvrage se révèle d'une très bonne qualité et comble un manque important concernant la compréhension des logiques du pouvoir islamiste soudanais et de son économie politique tout en présentant également un apport significatif à la littérature sur les manières de gouverner les ressources naturelles, et l'eau plus spécifiquement, dans un contexte international de changement climatique où la question de sa pénurie est particulièrement aiguë.

3 Le premier chapitre expose l'édifice conceptuel dans lequel s'inscrit l'ouvrage, à savoir un travail d'écologie politique dans lequel l'eau n'est pas conçue uniquement comme un phénomène physique mais également par un ensemble d'idées, de pratiques matérielles et de relations de pouvoir que l'auteur combine avec un travail classique de sociologie historique de l'État. Ce choix permet à l'auteur d'affirmer que l'environnement et le politique sont «co-constitutifs » et que les liens matériels et discursifs qui les unissent s'avèrent centraux pour saisir l'histoire moderne de l'État soudanais et d'une grande partie de la violence qui se déploie dans ses territoires.

4 Le deuxième chapitre s'emploie à développer cet argument en analysant la façon par laquelle les dirigeants de la vallée du Nil (Égypte comprise) ont conçu ce lien à travers l'histoire, à la fois d'un point de vue conceptuel mais également matériel. Il expose notamment les jeux des puissances coloniales autour du contrôle du Nil ou encore le développement de l'agriculture d'exportation durant le Soudan colonial en soulignant l'obsession scientiste d'une régulation optimum du fleuve qui passe par une centralisation de sa gestion et une certaine rationalisation. Le chapitre expose également longuement la stratégie développée par le gouvernement socialiste de Nimeiri qui a voulu faire du Soudan le grenier du monde arabe dans les années 1970 et 1980 et son cuisant échec. Verhoeven démontre par la suite combien ce projet a inspiré celui des islamistes.

5 Le troisième chapitre explique comment pour le régime d'Al-Ingaz l'islamisation de la société et sa modernisation sont les deux facettes d'une même pièce mais souligne également les paradoxes intrinsèques du projet que développent les islamistes dans la 
mesure où ils cherchent à attirer à eux deux groupes aux intérêts divergents : une petite bourgeoisie qui pourrait bénéficier de l'exclusion de l'élite attachée aux partis traditionnels, et les populations urbaines marginalisées. C'est notamment dans ce dernier groupe qu'il faudrait inclure les populations darfouriennes que nous évoquions au début de cette recension, mais le tableau brossé ici par Verhoeven reste très macro et omet dès lors certaines nuances. On comprend néanmoins comment, dans les années 1990, les disparités et inégalités territoriales s'accentuent et dans quelle mesure les choix de Tourabi en matière de politique étrangère contraignent drastiquement les possibilités de développement économique du pays.

6 Verhoeven montre ensuite comment ces choix jouent un rôle central dans la scission qui s'est opérée au sein des islamistes au tournant du millénaire. Le chapitre quatre s'attèle à décrire cette scission depuis l'intérieur opposant ceux favorables à l'ouverture-qui permettrait de mener à bien la «mission hydro-agricole »- et les plus rigoristes. Il faut noter ici combien l'analyse de Verhoeven donne la part belle aux stratégies et intentions des dirigeants et développe une idée partagée par de nombreux analystes du Soudan qui veut que le premier groupe sorti vainqueur de cette lutte interne assume pleinement une vision ethnicisée et élitiste de son action aux commandes de l'État. Pourtant, cette analyse occulte totalement le caractère souvent contingent de certains choix politiques. Verhoeven décrit le système opaque et autoritaire lié à la Dams Implementation Unit ayant pour principal, si ce n'est unique, but d'attribuer des contrats juteux aux proches du pouvoir tout en favorisant l'extraversion vers le Golfe.

7 Le cinquième chapitre s'intéresse quant à lui au contexte régional dans lequel le projet a pu se développer ; il revient notamment sur l'évolution des relations du Soudan avec l'Égypte et les pays du Golfe, et discute du partenariat stratégique avec la Chine. Verhoeven montre ici l'influence du modèle chinois d'État développeur que les dirigeants du Soudan semblent avoir troqué contre leurs prétentions religieuses et d'inclusion politique. Dans ce modèle, les dirigeants justifient leur position de pouvoir par leur capacité à transformer l'économie et par les bienfaits matériels qui en découlent.

8 Le dernier chapitre est un réquisitoire sans appel contre le modèle de gouvernement favorisé par cette «mission» et contre le contenu même de celle-ci. Verhoeven démontre efficacement à quel point les résultats, que l'on se place à un niveau macro ou micro, ne sont pas seulement décevants mais témoignent d'un amateurisme scientifique patent, d'une corruption extrême et d'un autoritarisme féroce. Verhoeven souligne combien ce système a nourri le fort mécontentement populaire qui s'est exprimé lors d'importantes manifestations en 2012-2013, mécontentement qui fait d'ailleurs écho à celui qui a conduit à la chute d'Omar Al-Bechir.

9 La conclusion de l'ouvrage revient efficacement sur les apports théoriques et empiriques du travail exposé et esquisse les pistes d'un futur programme de recherche qui interroge le modèle d'État développeur illibéral ailleurs en Afrique tout en ouvrant le débat sur les modèles de développement liés aux grands barrages dans le monde ou à la nouvelle géopolitique de l'eau en Afrique dans un contexte d'insécurité alimentaire et de changement climatique. 


\section{AUTEURS}

RAPHAËLLE CHEVRILLON-GUIBERT

IRD, UMR PRODIG, Paris 